\title{
Criptógamos do Parque Estadual das Fontes do Ipiranga, São Paulo, SP. Algas, 43: Zygnematophyceae (Mesotaeniaceae)
}

\author{
(10) Carlos E. de M. Bicudo ${ }^{1,2}$
}

Recebido: 17 julho 2018; aceito: 27 dezembro 2018

Como citar: Bicudo, C.E.M. 2019. Criptógamos do Parque Estadual das Fontes do Ipiranga, São Paulo, SP. Algas, 43: Zygnematophyceae. Hoehnea 46: e672018. http://dx.doi.org/10.1590/2236-8906-67/2018.

\begin{abstract}
Cryptogams of the Parque Estadual das Fontes do Ipiranga, São Paulo, SP. Algae, 43: Zygnematophyceae (Mesotaeniaceae). Paper is the taxonomic survey of the Mesotaeniaceae (Zygnemaphyceae, Zygnemales) present in the PEFI, Parque Estadual das Fontes do Ipiranga lacustrine systems. The examined material included periphyton and plankton from different aquatic systems within the PEFI, and the revision of all material cited for the Park deposited in the Scientific State Herbarium "Maria Eneyda P. Kauffmann Fidalgo" (SP). Five species were identified, which are distributed in three genera (Cylindrocystis, Mesotaenium, and Netrium). IAG Pond was the system presenting the highest species richness (three taxa) probably due to its oligotrophic characteristics.
\end{abstract}

Keywords: biodiversity, floristic survey, phytoplankton

RESUMO - (Cryptógamos do Parque Estadual das Fontes do Ipiranga, São Paulo, SP. Algas, 43: Zygnematophyceae (Mesotaeniaceae)). Trata do levantamento taxonômico das Mesotaeniaceae (Zygnemaphyceae, Zygnemales) de ambientes lacustres do PEFI, Parque Estadual das Fontes do Ipiranga. O material examinado proveio de coletas de material perifítico e planctônico em diferentes sistemas aquáticos do PEFI, além da revisão de todo material citado para o Parque e depositado no Herbário Científico do Estado "Maria Eneyda P. Kauffmann Fidalgo" (SP). Foram identificadas cinco espécies distribuídas em três gêneros (Cylindrocystis, Mesotaenium e Netrium). O Lago do IAG foi o ambiente que apresentou a maior riqueza específica (três táxons), provavelmente devido à característica oligotrófica do sistema.

Palavras-chave: biodiversidade, fitoplâncton, levantamento florístico

\section{Introdução}

A família Mesotaeniaceae está classificada na ordem Zygnemales (ou Zygnematales) da classe Zygnemaphyceae (ou Zygnematophyceae) (Lewis \& McCourt 2004). Estes autores revisaram os avanços mais significativos da sistemática das algas verdes publicados nos últimos, então, 20 anos. Os focos desses trabalhos foram táxons monofiléticos e uma visão mais ampla da filogenia e da evolução das algas verdes. Lewis \& McCourt (2004) procuraram avaliar, nesse trabalho, o tipo de informação que gerou tais mudanças e concluíram que muitas relações atualmente baseadas em biologia molecular jamais foram preditas ou previsíveis a partir de informação ultraestrutural.
Já foram realizados mais de 150 trabalhos sobre a taxonomia das microalgas de ambientes aquáticos do PEFI, Parque Estadual das Fontes do Ipiranga e mais de 60 sobre sua ecologia, porém, somente três registraram a presença de espécies de Mesotaeniaceae na referida área, sendo dois de material do Lago do IAG e um do Lago das Ninfeias.

Ferragut et al. (2005) documentaram a presença de Netrium digitus (Ehrenberg ex Ralfs) Itzigsohn \& Rothe var. lamellosum (Brébisson ex Kützing) Grönblad no Lago do IAG e Ferrari (2010) apenas relacionou, em uma tese de doutorado, a ocorrência de Netrium oblongum (De Bary) Lütkemüller var. cylindricum West \& West e Netrium sp. igualmente no Lago do IAG. Do último trabalho não constam descrição nem ilustração do material identificado.

1. Instituto de Botânica, Caixa Postal 68041, 04045-972 São Paulo, SP, Brasil

2 Autor para correspondência: cbicudo@terra.com.br 
Outra tese de doutorado, de Pereira (2013), também só relacionou a presença de Cylindrocystis brebissonii (Ralfs) De Bary e Mesotaenium macrococcum (Kützing ex Kützing) Roy \& Bisset no Lago das Ninfeias, sem descrever ou ilustrar os espécimes estudados.

Em se tratando de um levantamento florístico, o objetivo da atual contribuição foi realizar o inventário taxonômico das espécies de Mesotaeniaceae que ocorrem na área do PEFI visando, com isso, a contribuir para o conhecimento da biodiversidade das algas do referido Parque. Este trabalho faz parte do projeto maior intitulado "Criptógamos do Parque Estadual das Fontes do Ipiranga, São Paulo, SP, Algas", que já conta com 42 artigos publicados sobre a flórula dos diferentes grupos de algas e cianobactérias da área.

\section{Material e métodos}

Área de estudo - O estudo abordou sistemas aquáticos do PEFI, Parque Estadual das Fontes do Ipiranga situado na região sudeste do Município de São Paulo, entre os paralelos $23^{\circ} 38^{\prime}$ e $23^{\circ} 40^{\prime} \mathrm{S}$ e os meridianos $46^{\circ} 36^{\prime}$ e $46^{\circ} 38^{\prime} \mathrm{W}$.

Material - Foram coletados materiais perifítico e planctônico de sistemas aquáticos do PEFI e providenciada revisão de todo material citado para a referida Unidade de Conservação. Foram analisadas, enfim, amostras da coleção do Herbário Científico do Estado "Maria Eneyda P. Kauffmann Fidalgo" (SP) do Instituto de Botânica obtidas de diferentes "lagos", isto é, de pequenas represas localmente chamadas lagos como, por exemplo, o Lago das Ninfeias e o Lago do IAG em diferentes épocas do ano e ao longo das últimas cinco décadas.

Métodos - Os métodos gerais para o desenvolvimento do trabalho constam em Milanez et al. (1990).

O sistema de classificação utilizado foi de Lewis \& McCourt (2004). Para a identificação taxonômica foi utilizada bibliografia especifica, com destaque para os trabalhos de Prescott et al. (1972) e Croasdale \& Flint (1986).

A sequência dos gêneros dentro de cada família e das espécies dentro de seus respectivos gêneros seguiu simplesmente a ordem alfabética.

\section{Resultados e Discussão}

Os gêneros identificados no presente estudo estão classificados como segue:
Divisão Chlorophyta

Classe Zygnematophyceae

Ordem Zygnematales

Família Mesotaeniaceae

Mesotaenium

Cylindrocystis

Netrium

\section{Mesotaeniaceae Bohlin}

Indivíduos unicelulares isolados ou, muito raro, formando pares devido ao processo recente de divisão celular. No último caso, são envoltos pela parede gelatinizada da célula-mãe. A parede celular é formada por uma peça única e não apresenta constrição mediana e, via-de-regra, é lisa, mas, ocasionalmente pode apresentar poros diminutos; ainda mais raro é apresentar diminutos espinhos pontiagudos, pelos ou grânulos. O cloroplastídio pode ser único ou existirem dois por célula; e é axial, laminado e apresenta um a vários pirenoides. A conjugação nesta família ocorre através de um tubo formado entre duas células, com o protoplasma de cada uma transformado em gameta. A meiose ocorre durante a germinação do zigósporo. Certas vezes, há formação de dois gametas por célula e a produção, consequente, de dois zigotos em cada célula.

Chave para identificação dos gêneros estudados

1. Cloroplastídio laminar Mesotaenium

1. Cloroplastídio estrelado ou com um eixo principal do qual irradiam lâminas verticais

2. Cloroplastídio estrelado ................. Cylindrocystis

2. Cloroplastídio com um eixo principal do qual irradiam projeções laminares verticais, de bordo sucessivamente reentrante e saliente .. Netrium

Cylindrocystis Lemmermann 1898.

Célula cilíndrica ou quase, reta ou levemente arqueada, com os polos amplamente arredondados e, em geral, destituída de constrição mediana; mais raro, entretanto, é possuir uma pequena reentrância mediana rasa, em forma de ângulo agudo. A parede celular é sempre lisa, constituída por uma peça única e pode ser hialina ou tingida de tons que variam do amarelado ao acastanhado dependendo da quantidade de impregnação com sais de ferro. Há dois cloroplastídios em cada célula situados axialmente, cuja forma é esteloide ou muito próximo. Cada cloroplastídio possui um pirenoide globoso ou bastoniforme localizado mais ou menos centralmente. 
A aparente raridade de notícias sobre a ocorrência de Mesotaeniaceae no Estado de São Paulo é devida à coleta em ambientes não propícios à sua existência. Estas algas são planctônicas, mas preferem ambiente ácido. É comum formarem massas gelatinosas em meio à vegetação da zona litorânea de ambientes aquáticos ou sobre o solo de barrancos constantemente umedecidos.

Apenas uma espécie identificada:

Cylindrocystis brebissonii Meneghini ex De Bary var. brebissonii, Untersuchungen über die Familie der Conjugaten (Zygnemaceen und Desmidiaceen). 35, pl. 7, fig. E 1-22. 1858.

Figura 1

Célula isolada, cilíndrica, 2-4 vezes mais longa que larga, não constrita na parte média, polos amplamente arredondados, 26-85,4 $\mu \mathrm{m}$ compr., 10-23 um diâm., cloroplastídios 2, axiais, usualmente com alguns poucos prolongamentos radiais, pirenoide 1 em cada plastídio, central, usualmente grande, suco celular em geral avermelhado ou arroxeado.

Material examinado: Lago das Ninfeias, sem maior informação.

Hábitat: plâncton.

Distribuição no PEFI: Lago das Ninfeias (Pereira 2013).

\section{Mesotaenium Nageli 1849.}

Indivíduo isolado ou que pode ocorrer junto a outro formando pares e assim permanecer por certo tempo logo apos o processo de divisão celular. A célula é cilíndrica, em geral reta, raramente pouco arqueada na região mediana e possui os polos amplamente arredondados ou arredondado-truncados. A parede celular é constituída por uma peça única e é sempre lisa. O mais corriqueiro é encontrar um cloroplastídio por célula, mas também podem ocorrer dois, sendo um em cada semicélula. Os cloroplastídios podem ser laminares, retos ou torcidos em hélice, ter o bordo liso ou denteado e ocupam situação axial na célula na maioria das vezes, raro parietal. Possuem desde um até vários pirenoides arranjados em uma linha longitudinal mediana no plastídio.

As espécies deste gênero ocorrem dominantemente em ambientes subaéreos (atmofíticos) como solo, rocha ou córtex de árvores, formando massas mucilaginosas macroscópicas de cor castanho ou misturadas com briófitas.
Apenas uma espécie identificada:

Mesotaenium macrococcum (Kützing ex Kützing) Roy \& Bisset var. macrococcum, Annals of Scottish Natural History 1894(9): 253. 1894.

Figura 2

Célula isolada, cilíndrica, 2-2,5 vezes mais longa que larga, polos arredondado-trucados,

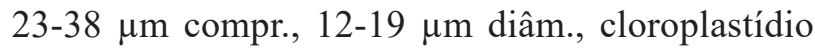
1, axial, laminar, margem denteada, pirenoide 1 , aproximadamente central.

Material examinado: Lago das Ninfeias, sem maior informação.

Hábitat: plâncton.

Distribuição no PEFI: Lago das Ninfeias (Pereira 2013).

Netrium (Nägeli) Itzigsohn \& Rothe emend. Lütkemuller emend. Gontcharov \& Melkonian 2010 .

Indivíduos unicelulares isolados. A célula é elíptica, elíptico-fusiforme ou mais ou menos cilíndrica, pelo menos duas e até $20-25$ vezes mais longa que larga. É destituída de constrição mediana e os polos são amplamente arredondados a arredondadotruncados. A parede celular é lisa e constituída por uma peça única. Ocorrem dois cloroplastídios axiais do tipo esteloide em cada célula, com seis a 12 expansões laterais, radiais, que partem de um eixo comum, cujo bordo é sucessivamente reentrante e saliente. $\mathrm{O}$ pirenoide pode ser esférico ou em forma de bastão curto.

Gontcharov \& Melkonian (2010) sequenciaram 53 linhagens de algas verdes conjugantes, incluindo material de Roya, Netrium, Cylindrocystis e Mesotaenium e observaram que as espécies de Netrium distribuíram-se em três ramos da árvore filogenética dependendo (1) do número de plastídios por célula, (2) da morfologia superficial do plastídio, isto é, da estrutura e do arranjo das lamelas radiais e (3) da posição do núcleo na célula ou de seu comportamento durante a divisão celular. A partir desses resultados, os referidos autores propuseram o gênero Nucleotaenium e ressuscitaram o gênero Planotaenium.

Chave para identificação das espécies

1. Célula elíptico-alongada ... N. digitus var. lamellosum 1. Célula cilíndrica ........... N. oblongum var. cylindricum 

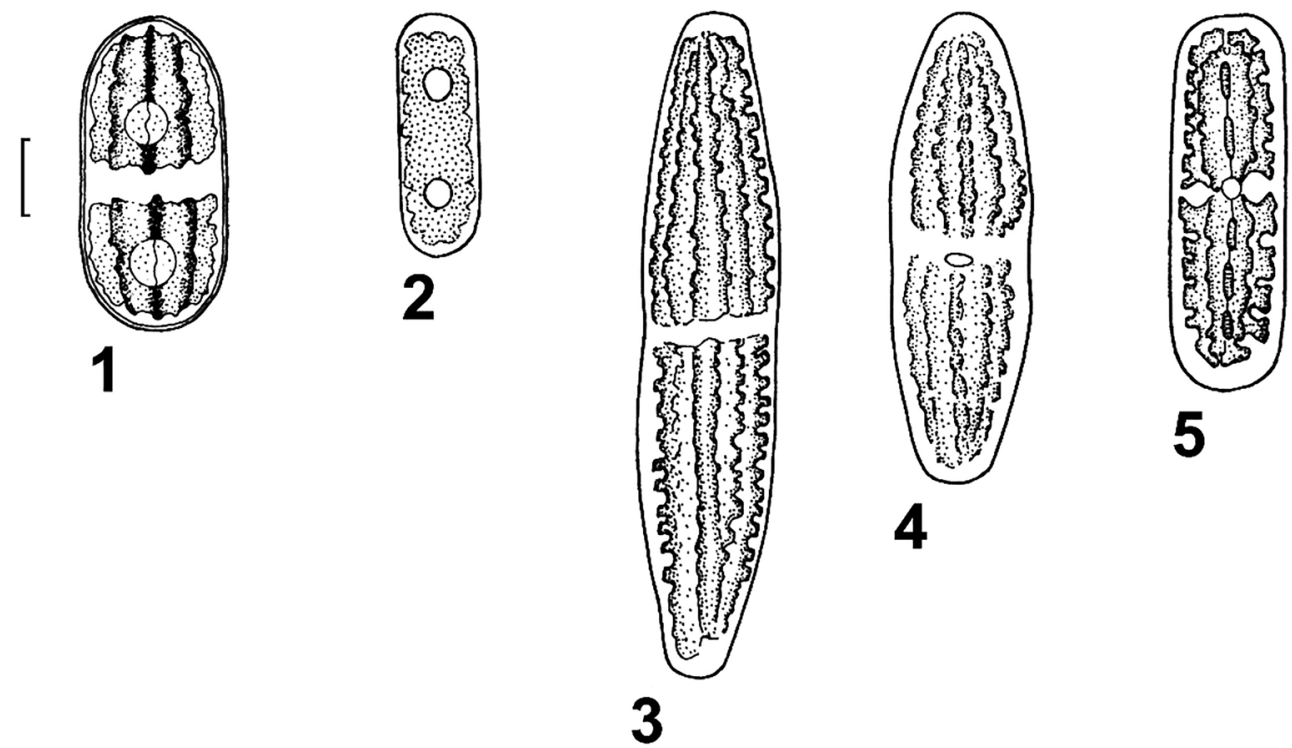

Figuras 1-5: Mesotaeniaceae do PEFI: 1. Cylindrocystis brebissonii Meneghini ex De Bary var. brebissonii (segundo Bicudo \& Bicudo 1970). 2. Mesotaenium macrococcum (Kützing ex Kützing) Roy \& Bisset var. macrococcum (segundo Bicudo 1969). 3-4. Netrium digitus (Ehrenberg ex Ralfs) Itzigsohn \& Rothe var. lamellosum (Brébisson ex Kützing) Grönblad (segundo Prescott et al. 1972). 5. N. oblongum (De Bary) Lütkemüller var. cylindricum West \& West (segundo Prescott et al. 1972). Escala $=10 \mu \mathrm{m}$.

Figures 1-5: PEFI Mesotaeniaceae. 1. Cylindrocystis brebissonii Meneghini ex De Bary var. brebissonii (from Bicudo \& Bicudo 1970). 2. Mesotaenium macrococcum (Kützing ex Kützing) Roy \& Bisset var. macrococcum (from Bicudo 1969). 3-4. Netrium digitus (Ehrenberg ex Ralfs) Itzigsohn \& Rothe var. lamellosum (Brébisson ex Kützing) Grönblad (from Prescott et al. 1972). 5. N. oblongum (De Bary) Lütkemüller var. cylindricum West \& West (from Prescott et al. 1972). Scale bar $=10 \mu \mathrm{m}$.

Netrium digitus (Ehrenberg ex Ralfs) Itzigsohn \& Rothe var. lamellosum (Brébisson ex Kützing) Grönblad, Acta Societatis pro Fauna etFlora Fennica 47(4): 13. $1920 \equiv$ Penium lamellosum Brébisson, Memories de la Société des Sciences Naturelles de Cherbourg 4: 146, pl. 2, fig. 34. 1856.

Figuras 3-4

Célula elíptico-alongada, lados pouco retusos na porção mediana, polos arredondado-truncados, 12,8-16 $\mu$ m compr., 8,8-10,4 $\mu$ m diâm., cloroplastídio 1 por semicélula, axial, 6-10 expansões laterais que partem do eixo central, cujo bordo é sucessivamente reentrante e saliente.

Material examinado: Lago do IAG, sem maior informação.

Hábitat: plâncton.

Distribuição no PEFI: Lago do IAG (Ferragut et al. 2005, Lopes 1999).
Netrium oblongum (De Bary) Lütkemüller var. cylindricum West \& West, Journal of Botany 41: 40, pl. 446, fig. 10. 1903 EPenium oblongum De Bary in Wolle, Desmids of the United States and list of American Pediastrums. 35, pl. 5, fig. 17. 1892.

Figura 5

Célula cilíndrica, polos amplamente arredondados, ca. $18 \mu \mathrm{m}$ compr., ca. $12 \mu \mathrm{m}$ diâm., cloroplastídio 1 , axial, 5-10 expansões laterais radiais que partem do eixo central, cujo bordo é sucessivamente reentrante e saliente.

Material examinado: Lago do IAG, sem maior informação.

Hábitat: plâncton.

Distribuição no PEFI: Lago do IAG (Ferrari 2010).

\section{Netrium sp.}

Ferrari (2010) identificou alguns espécimes do gênero sem, entretanto, chegar à espécie que representavam. O nome aparece na lista dos materiais que identificou sem, porém, descrição nem ilustração. 
Hábitat: perifíton.

Distribuição no PEFI: Lago do IAG (Ferrari 2010).

Chave geral

1. Cloroplastídio laminar .... Mesotaenium macrococcum var. macrococcum

1. Cloroplastídio estrelado ou com um eixo principal do qual irradiam lâminas verticais

2. Cloroplastídio estrelado

Cylindrocystis brebissonii var. brebissonii

2. Cloroplastídio com um eixo principal do qual irradiam projeções laminares verticais de bordo sucessivamente reentrante e saliente

(Netrium)

3. Célula elíptico-alongada N. digitus var. lamellosum

3. Célula cilíndrica

N. oblongum var. cylindricum

\section{Agradecimentos}

Agradeço ao CNPq, Conselho Nacional de Desenvolvimento Científico e Tecnológico, por Bolsa de Produtividade (Proc. No 309474/2010-8).

\section{Literatura citada}

Croasdale, H. \& Flint, E.A. 1986. Flora of New Zealand: freshwater algae, Chlorophyta, Desmids with ecological comments on their habitats. V.R. Ward Government Printer, Wellington.
Ferrari, F. 2010. Estrutura e dinâmica da comunidade de algas planctônicas e perifíticas (com ênfase nas diatomáceas) em reservatórios oligotrófico e hipertrófico (Parque Estadual das Fontes do Ipiranga, São Paulo). Tese de Doutorado, Universidade Estadual Paulista, Rio Claro.

Ferragut, C., Lopes, M.R.M., Bicudo, D.C., Bicudo, C.E.M. \& Vercellino, I.S. 2005. Ficoflórula perifítica e planctônica (exceto Bacillariophyceae) de um reservatório oligotrófico raso (Lago do IAG, São Paulo). Hoehnea 32: 137-184.

Lewis, L.A. \& McCourt, R.M. 2004. Green algae and the origin of land plants. American Journal of Botany 91: 1535-1556.

Lopes, M.R.M. 1999. Eventos perturbatórios que afetam a biomassa, a composição e a diversidade de espécies do fitoplâncton em um lago tropical oligotrófico raso (Lago do Instituto Astronômico e Geofísico, São Paulo, SP). Tese de Doutorado, Universidade de São Paulo, São Paulo.

Milanez, A.L., Bicudo, C.E.M., Vital, D.M. \& Grandi, R.A.P. 1990. Criptógamos do Parque Estadual das Fontes do Ipiranga, São Paulo, SP: planejamento. Hoehnea 17: 43-49.

Pereira, J.S. 2013. Estrutura e dinâmica da comunidade fitoplanctônica no período de cinco anos em ambiente mesotrófico (Lago das Ninfeias), Parque Estadual das Fontes do Ipiranga, São Paulo. Tese de Doutorado, Universidade Estadual Paulista, Rio Claro.

Prescott, G.W., Croasdale, H.T. \& Vinyard, W.C. 1972. North American Flora: Desmidiales, Saccodermae, Mesotaeniaceae. The New York Botanical Garden, New York. 